TRANSACTIONS OF THE

AMERICAN MATHEMATICAL SOCIETY

Volume 358, Number 11, November 2006, Pages 4749-4764

S 0002-9947(06)04088-8

Article electronically published on April 17, 2006

\title{
A HUREWICZ-TYPE THEOREM FOR ASYMPTOTIC DIMENSION AND APPLICATIONS TO GEOMETRIC GROUP THEORY
}

\author{
G. C. BELL AND A. N. DRANISHNIKOV
}

\begin{abstract}
We prove an asymptotic analog of the classical Hurewicz theorem on mappings that lower dimension. This theorem allows us to find sharp upper bound estimates for the asymptotic dimension of groups acting on finitedimensional metric spaces and allows us to prove a useful extension theorem for asymptotic dimension. As applications we find upper bound estimates for the asymptotic dimension of nilpotent and polycyclic groups in terms of their Hirsch length. We are also able to improve the known upper bounds on the asymptotic dimension of fundamental groups of complexes of groups, amalgamated free products and the hyperbolization of metric spaces possessing the Higson property.
\end{abstract}

\section{INTRODUCTION}

In classical dimension theory the Hurewicz theorem on mappings that lower dimension is a powerful tool. One statement of the theorem is the following (see [1], for example).

Theorem. Let $X$ and $Y$ be compact metric spaces and $f: X \rightarrow Y$ a continuous map. Suppose that there is some $n$ so that for every $y \in Y, \operatorname{dim} f^{-1}(y) \leq n$. Then $\operatorname{dim} X \leq \operatorname{dim} Y+n$.

Gromov defined the asymptotic dimension of a metric space in his study of asymptotic invariants of finitely generated groups in 12. The asymptotic dimension of a metric space $X$, asdim $X$, is defined to be the smallest integer $n$ so that for every $R$ there is a uniformly bounded cover of $X$ so that no $R$-ball in $X$ meets more than $n+1$ elements of the cover.

Asymptotic dimension is a coarse invariant (see [14]), so in particular it is a quasi-isometry invariant. Much of the interest in asymptotic dimension has been directed at showing that certain classes of groups have finite asymptotic dimension in a natural metric; see for example, [1, [2], 9], [12, [13. For the most part we are concerned with finitely generated groups in this note. The metric we associate to a finitely generated group is the word metric coming from some finite generating set. As any two such metrics are Lipschitz equivalent, asdim $\Gamma$ is well defined without reference to a generating set. Finite asdim results for groups became important

Received by the editors July 20, 2004.

2000 Mathematics Subject Classification. Primary 20F69, 20F65; Secondary 20E08, 20E06.

Key words and phrases. Asymptotic dimension, free products, nilpotent groups.

The second author was partially supported by NSF Grant DMS-0305152.

(C)2006 American Mathematical Society 
following a theorem of $\mathrm{Yu}$ [15, which showed that the Novikov higher signature conjecture holds for $\Gamma$ with asdim $\Gamma<\infty$. It is because of Yu's theorem that many results were aimed only at showing that asdim $\Gamma$ was finite and not concerned with computing the exact dimension.

There are analogies between local topology and asymptotic topology as well as "dictionaries" translating between the local and asymptotic worlds; see [7. For dimension, however, the correspondence is often not direct. For example, in classical dimension theory we have the Urysohn-Menger Theorem giving the sharp upper bound: $\operatorname{dim}(X \cup Y) \leq \operatorname{dim} X+\operatorname{dim} Y+1$. In the asymptotic case, by contrast, the authors showed in [2] (also, see section 4) that $\operatorname{asdim}(X \cup Y)=$ $\max \{\operatorname{asdim} X, \operatorname{asdim} Y\}$. Also, $\bigcup_{i=1}^{\infty} \operatorname{dim} F_{i}=\max \left\{\operatorname{dim} F_{i}\right\}$ for closed sets, but not every finitely generated group $\Gamma$ has asdim $\Gamma=0$, so there cannot be a direct analog of the countable union theorem. In section 4 we state a countable union theorem for asdim from 2 .

In contrast with the union theorems, the asymptotic analog of the Hurewicz theorem on mappings that lower dimension is very much a direct analog of its classical counterpart. In the third section we prove our version of an asymptotic Hurewicz theorem.

Theorem 1, Let $f: X \rightarrow Y$ be a Lipschitz map of a geodesic metric space to a metric space. Suppose that for every $R>0,\left\{f^{-1}\left(B_{R}(y)\right)\right\}_{y \in Y}$ satisfies the inequality asdim $\leq n$ uniformly (see section 2 ). Then $\operatorname{asdim} X \leq \operatorname{asdim} Y+n$.

This leads to an upper bound estimate for finitely generated groups acting on finite-dimensional metric spaces that agrees with the formula conjectured in [2] as well as in 14.

The fourth section is devoted to a specialized version of the Hurewicz theorem. When the codomain of the Lipschitz map is a tree and some asymptotic disjointness conditions are satisfied, the estimate on the asymptotic dimension can be improved. This leads to a generalization of the formula for the asymptotic dimension of a free product of groups. In particular, we consider a free product of pointed metric spaces and compute their asymptotic dimension. This also leads to an upper bound estimate on the asymptotic dimension of free products with amalgamation that, unfortunately, is given in terms of the asymptotic dimension of quotients. Whereas asdim is monotonic in subsets, the asdim of a quotient cannot be determined from the asdim of the spaces in the quotient (see the remarks following Theorem 6).

In the final section we apply the Hurewicz theorem to finitely generated groups acting by isometries on metric spaces. This leads us to an extension theorem for asdim.

Theorem 7, Let $\phi: G \rightarrow H$ be a surjection of a finitely generated group with $\operatorname{ker} \phi=K$. Then, $\operatorname{asdim} G \leq \operatorname{asdim} H+\operatorname{asdim} K$.

We conclude the paper with applications of our extension theorem to nilpotent groups, some amalgamated free products and the hyperbolization of metric spaces possessing the Higson property.

\section{AsYmptotic DIMENSION AND UNIFORM MAPPING CYLINDERS}

As mentioned in the Introduction, Gromov defined the asdim of a metric space in [12. There are many equivalent formulations of asdim. As we will need to 
pass between them, we summarize some of the equivalences below. (All of these equivalences are stated in [12]; for explicit proofs, see [3], 14].)

Theorem. For a metric space $X$ the following are equivalent:

(1) for every $D>0$ there exist $D$-disjoint families $\mathcal{U}_{0}, \ldots, \mathcal{U}_{n}$ of uniformly bounded sets whose union covers $X$;

(2) for every $R>0$ there exists a uniformly bounded cover $\mathcal{U}$ of $X$ in which no $R$-ball in $X$ meets more than $n+1$ elements of the cover $\mathcal{U}$;

(3) for every $L>0$ there exists a uniformly bounded cover of $X$ with multiplicity $\leq n+1$ and with Lebesgue number $>L$;

(4) for every $\epsilon>0$ there is a uniformly cobounded, $\epsilon$-Lipschitz map $\phi: X \rightarrow K$ to a uniform polyhedron of dimension $\leq n$.

Recall that a family $\mathcal{U}$ of subsets of a metric space is said to be $D$-disjoint if $d(U, V)>D$ for every $U \neq V$ in $\mathcal{U}$. The condition on the cover in item (2) Of The Theorem is often referred to as $R$-multiplicity $\leq n+1$. The Lebesgue number of a cover $\mathcal{U}$ of a metric space $X$ is $L(\mathcal{U})=\inf \{\max \{d(x, X \backslash U) \mid U \in \mathcal{U}\} \mid x \in X\}$. A uniform polyhedron is the geometric realization of a simplicial complex in $\ell^{2}$, with the metric it inherits as a subset. Finally, a map $\phi$ to a uniform polyhedron is uniformly cobounded if there is a number $B$ so that $\operatorname{diam}\left(\phi^{-1}(\sigma)\right) \leq B$ for all simplices $\sigma$.

Definition. A metric space $X$ has $\operatorname{asdim} X \leq n$ if it satisfies any of the equivalent conditions of the previous Theorem.

Often we will need to work with the canonical projection of a cover to its nerve. In fact, the implication $(3) \Longrightarrow(4)$ of the previous theorem can be seen by simply applying the canonical projection to the nerve. Let $\mathcal{U}$ be an open cover of a metric space $X$. The canonical projection to the nerve $p: X \rightarrow N$ erve $(\mathcal{U})$ is defined by the partition of unity $\left\{\phi_{U}: X \rightarrow \mathbb{R}\right\}_{U \in \mathcal{U}}$, where $\phi_{U}(x)=d(x, X \backslash U) / \sum_{V \in \mathcal{U}} d(x, X \backslash V)$. The family $\left\{\phi_{U}: X \rightarrow \mathbb{R}\right\}_{U \in \mathcal{U}}$ defines a map $p$ to the Hilbert space $\ell^{2}(\mathcal{U})$ with basis indexed by $\mathcal{U}$. The nerve Nerve $(\mathcal{U})$ of the cover $\mathcal{U}$ is realized in $\ell^{2}(\mathcal{U})$ by taking every vertex $U$ to the corresponding element of the basis. Clearly, the image of $p$ lies in the nerve.

In 3 the authors showed that the canonical projection $p: \mathcal{U} \rightarrow$ Nerve $(\mathcal{U})$ of a cover $\mathcal{U}$ with multiplicity $k+1$ and Lebesgue number $L$ is $\frac{(2 k+3)^{2}}{L}$-Lipschitz (cf. Proposition 77).

In the statement of the asymptotic Hurewicz theorem we need the following natural notion of uniformity for asdim defined by the authors in 2. A family $\left\{X_{\alpha}\right\}$ of subsets of a metric space $X$ satisfies the inequality asdim $X_{\alpha} \leq n$ uniformly (see [3]) if for large $D>0$ there is an $R>0$ such that there exist $R$-bounded, $D$ disjoint families $\mathcal{U}_{\alpha}^{0}, \ldots, \mathcal{U}_{\alpha}^{n}$ so that $\bigcup_{i=0}^{n} \mathcal{U}_{\alpha}^{i}$ is a cover of each $X_{\alpha}$. A basic example of families satisfying asdim $X_{\alpha} \leq n$ uniformly is when all the families are isometric.

In view of the fact that any tree $T$ has $\operatorname{asdim} T \leq 1$ (see [14]), the authors proved in [3] what could be called a first approximation to the asymptotic Hurewicz theorem. In particular, the main result was

Theorem ([3, Theorem 1]). Suppose that the finitely generated group $\Gamma$ acts cocompactly by isometries on a tree $X$. Then, $\operatorname{asdim} \Gamma \leq k+1$, where $\operatorname{asdim} \Gamma_{x} \leq k$ for all stabilizers $\Gamma_{x}$ of vertices $x \in X$. 
The proof used the characterization of asymptotic dimension in terms of uniformly cobounded, Lipschitz maps to uniform polyhedra. The argument here is similar and relies heavily on the notion of simplicial mapping cylinders. We summarize the pertinent results on simplicial mapping cylinders in the following proposition.

Proposition 1 ([3, Propositions 2, 3]). For every simplicial map $f: X \rightarrow Y$ from a n-dimensional simplicial complex $X$, the mapping cylinder $M_{f}$ admits a triangulation with the set of vertices equal to the disjoint union of vertices of $X$ and $Y$; there is a constant $c_{n}$ so that the quotient map $q: X \times[0,1] \rightarrow M_{f}$ is $c_{n}$-Lipschitz, where $M_{f}$ is given the uniform metric it inherits from $\ell^{2}$.

Proposition 2 ([3, Proposition 4]). Let $A \subset W \subset X$ be subsets in a geodesic metric space $X$ such that the $r$-neighborhood $N_{r}(A)$ is contained in $W$ and let $f: W \rightarrow Y$ be a continuous map to a metric space $Y$. Assume that the restrictions $\left.f\right|_{N_{r}(A)}$ and $\left.f\right|_{W \backslash N_{r}(A)}$ are $\epsilon$-Lipschitz. Then $f$ is $\epsilon$-Lipschitz.

We end this section with a computation we will need later.

Proposition 3. Let $X, Y$ and $Z$ be metric spaces. Suppose $f: X \rightarrow Y$ and $g: X \rightarrow Z$ are Lipschitz functions with Lipschitz constants $\lambda_{f}$ and $\lambda_{g}$, respectively. Then, the map $f \times g: X \rightarrow Y \times Z$ defined by $x \mapsto(f(x), g(x))$ is $\sqrt{2} \max \left\{\lambda_{f}, \lambda_{g}\right\}$ Lipschitz in the product metric $\sqrt{d_{Y}^{2}+d_{Z}^{2}}$

Proof. The proof is an elementary calculation:

$$
\begin{aligned}
d_{Y \times Z}\left((f(x), g(x)),\left(f\left(x^{\prime}\right), g\left(x^{\prime}\right)\right)\right) & =\sqrt{\left[d_{Y}\left(f(x), f\left(x^{\prime}\right)\right)\right]^{2}+\left[d_{Z}\left(g(x), g\left(x^{\prime}\right)\right)\right]^{2}} \\
& \leq \sqrt{\left(\lambda_{f}^{2}+\lambda_{g}^{2}\right) d_{X}\left(x, x^{\prime}\right)^{2}} \\
& \leq \sqrt{2} \max \left\{\lambda_{f}, \lambda_{g}\right\} d_{X}\left(x, x^{\prime}\right) .
\end{aligned}
$$

\section{An Asymptotic Hurewicz theorem}

We need a version of Lemma 1 from [3] (see also our Lemma 3). The result is very technical, so we break it up over the next two lemmas and one proposition.

Lemma 1. Let $f: X \rightarrow Y$ be a $\lambda$-Lipschitz map of a geodesic metric space to a metric space with $\lambda \geq 1$. Let $r>1$ be given and suppose that $\mathcal{W}$ is a uniformly bounded cover of $Y$ with uniformly bounded $\lambda r$-multiplicity. Let $\tau$ be a simplex in Nerve $\left(N_{\lambda r}(\mathcal{W})\right)$ maximal with respect to containment, and take $\tau^{\prime}$ to be a simplex in $\beta^{1} \tau$ with $d=\operatorname{dim} \tau=\operatorname{dim} \tau^{\prime}$. For $i=0, \ldots, k$, let $W_{i}$ denote the vertex of $\tau^{\prime}$ corresponding to an $i$-face of $\tau$. Put $X_{\tau^{\prime}}=f^{-1}\left(\bigcup_{i=0}^{k} W_{i}\right)$. Finally suppose that there exist families $\mathcal{U}_{0}, \ldots, \mathcal{U}_{k}$ of uniformly bounded sets with multiplicities $\leq n+1$ such that

(1) $\mathcal{U}_{i}$ covers $f^{-1}\left(\bigcup_{j=0}^{i} W_{i}\right)$ and

(2) for all $i<j$ there exist simplicial maps $\psi_{(i)}^{(j)}: \operatorname{Nerve}\left(\mathcal{U}_{i}\right) \rightarrow \operatorname{Nerve}\left(\mathcal{U}_{j}\right)$.

Then there exists a uniformly cobounded, Lipschitz map $\phi: X_{\tau^{\prime}} \rightarrow K_{\tau^{\prime}}$ to a uniform polyhedron of dimension $n+k$.

Remark. The existence of such a map is not difficult to see through use of the finite union theorem from [2] and the fourth definition of asdim given above, but we will need specific properties of the map we construct here. 
Proof. For $x \in X_{\tau^{\prime}}$, define

$$
t_{i}(x)=\max \left\{0, \frac{\lambda r-\operatorname{dist}\left(f(x), W_{i}\right)}{\lambda r}\right\} .
$$

Observe that $0 \leq t_{i}(x) \leq 1$ with $t_{i}(x)=1$ precisely when $f(x) \in W_{i}$, and $t_{i}(x)>0$ if and only if $f(x)$ is in the interior of $N_{\lambda r}\left(W_{i}\right)$. Also, observe that on $X_{\tau^{\prime}}, t_{0} \equiv 1$.

We define the map $\phi: X_{\tau^{\prime}} \rightarrow K_{\tau^{\prime}}$ as a combination of simpler maps. First, we define $\phi_{0}: X_{\tau^{\prime}} \rightarrow \operatorname{Nerve}\left(\mathcal{U}_{0}\right)$ by $\phi_{0}(x)=p_{\mathcal{U}_{0}}(x)$, where $p_{\mathcal{U}_{0}}$ denotes the canonical projection to the nerve $\operatorname{Nerve}\left(\mathcal{U}_{0}\right)$. We define $\phi_{1}$ before passing to a general description of $\phi_{i}$.

First, put $g_{1}=\psi_{(0)}^{(1)}: \operatorname{Nerve}\left(\mathcal{U}_{0}\right) \rightarrow \operatorname{Nerve}\left(\mathcal{U}_{1}\right)$. Let $M_{g_{1}}$ denote the uniform mapping cylinder and let $q_{1}: \operatorname{Nerve}\left(\mathcal{U}_{0}\right) \times[0,1] \sqcup N e r v e\left(\mathcal{U}_{1}\right) \rightarrow M_{g_{1}}$ be the quotient and uniformization map.

Define $\phi_{1}: X_{\tau^{\prime}} \rightarrow M_{g_{1}}$ by

$$
\phi_{1}(x)= \begin{cases}q_{1}\left(\phi_{0}(x), 2 t_{1}(x)\right), & \text { if } t_{1}(x) \in\left[0, \frac{1}{2}\right], \\ 2\left(1-t_{1}(x)\right) \psi_{(0)}^{(1)} \phi_{0}(x)+\left(2 t_{1}(x)-1\right) p_{\mathcal{U}_{1}}(x), & \text { otherwise. }\end{cases}
$$

More generally, suppose that $\phi_{p-1}$ and $g_{p-1}$ have been defined. Define $g_{p}$ : $M_{g_{p-1}} \rightarrow \operatorname{Nerve}\left(\mathcal{U}_{p}\right)$ by

$$
\begin{aligned}
& g_{p}\left(\left[z, t_{1}, \ldots, t_{p-1}\right]\right) \\
& \quad=t_{p-1} \psi_{(p-1)}^{(p)} \psi_{(0)}^{(p-1)}(z)+\left(1-t_{p-1}\right)\left[t_{p-2} \psi_{(p-2)}^{(p)} \psi_{(0)}^{(p-2)}(z)+\left(1-t_{p-2}\right)[\cdots]\right] .
\end{aligned}
$$

Here we have extended the $\psi$ by defining $\psi_{(0)}^{(j)}(z)=z$ for all $z \in \operatorname{Nerve}\left(\mathcal{U}_{j}\right)$.

Next, put

$$
\phi_{p}(x)= \begin{cases}q_{p}\left(\phi_{p-1}(x), 2 t_{p}(x)\right), & \text { if } t_{p}(x) \in\left[0, \frac{1}{2}\right], \\ 2\left(1-t_{p}(x)\right) \psi_{(p-1)}^{(p)} \phi_{p-1}(x)+\left(2 t_{p}(x)-1\right) p_{\mathcal{U}_{p}}(x), & \text { otherwise }\end{cases}
$$

where, as before, $q_{p}$ is the uniformization and quotient map to the mapping cylinder of $M_{g_{p}}$. Put $\phi: X_{\tau^{\prime}} \rightarrow K_{\tau^{\prime}}$ equal to $\phi_{k}$.

First we show that $\phi$ is uniformly cobounded. To this end, let $\sigma \in K_{\tau^{\prime}}$ be a simplex. Suppose that $\xi, \eta \in \sigma$ and that $x_{\xi} \mapsto \xi, x_{\eta} \mapsto \eta$ under $\phi$. Then, $p_{\mathcal{U}_{k}}\left(x_{\xi}\right)$ and $p_{\mathcal{U}_{k}}\left(x_{\eta}\right)$ lie in the same simplex and obviously $f\left(x_{\xi}\right)$ and $f\left(x_{\eta}\right)$ lie in the same simplex. Thus $\operatorname{dist}\left(x_{\xi}, x_{\eta}\right) \leq \max \left\{2 b\left(\mathcal{U}_{i}\right), 2 b(\mathcal{W})\right\}$, which is a uniform bound. (Here $b\left(\mathcal{U}_{i}\right)$ denotes an upper bound on the diameters of the sets in $\left.\mathcal{U}_{i}.\right)$

It remains to show that the map $\phi$ is Lipschitz and to compute the Lipschitz constant. We consider $\phi: X_{\tau^{\prime}} \rightarrow M_{k}$. Observe that $x \in N_{\lambda r / 2}\left(W_{k}\right)$ if and only if $\frac{1}{2} \leq t_{k}(x) \leq 1$. So, applying Proposition 2, we see that $\phi_{k}$ is Lipschitz if it is Lipschitz when $t_{k}(x) \in\left[0, \frac{1}{2}\right]$ and when $t_{k}(x) \in\left[\frac{1}{2}, 1\right]$. (Here we are using the fact that $X$ is geodesic.) But, the definitions of these maps depend on $\phi_{k-1}$, which in turn depend on $\phi_{k-2}$. Thus, we begin with $\phi_{0}$ and work up inductively.

The map $\phi_{0}$ is just $p_{\mathcal{U}_{0}}$, so by [3, Proposition 1] it is $\frac{(2 n+3)^{2}}{L\left(\mathcal{U}_{0}\right)}-$ Lipschitz, where $L\left(\mathcal{U}_{0}\right)$ is the Lebesgue number of $\mathcal{U}_{0}$. Next, we consider $\phi_{1}$. We recall the definition

$$
\phi_{1}(x)= \begin{cases}q_{1}\left(\phi_{0}(x), 2 t_{1}(x)\right), & \text { if } t_{1}(x) \in\left[0, \frac{1}{2}\right], \\ 2\left(1-t_{1}(x)\right) \psi_{(0)}^{(1)} \phi_{0}(x)+\left(2 t_{1}(x)-1\right) p_{\mathcal{U}_{1}}(x), & \text { otherwise. }\end{cases}
$$


Note that in the second case, we have $\operatorname{dist}\left(x, f^{-1}\left(W_{1}\right)\right) \leq \frac{\lambda r}{2}$, so by Proposition 2 it suffices to show that the map is Lipschitz in both cases; then $\phi_{1}$ will be Lipschitz with constant equal to the max of the constants from each of the cases.

In the first case, we know that $\phi_{0}$ is $\frac{(2 n+3)^{2}}{L\left(\mathcal{U}_{0}\right)}$-Lipschitz, $t_{1}(x)$ is $\frac{2}{\lambda r}$-Lipschitz, and $q_{1}$ is $c_{n}$-Lipschitz. Thus, the map $\phi_{1}$ is $c_{n} \sqrt{2}\left(\max \left\{\frac{(2 n+3)^{2}}{L\left(\mathcal{U}_{0}\right)}, \frac{2}{\lambda r}\right\}\right)$-Lipschitz, by Proposition 3. In the second case, we apply the Leibnitz rule to see that the sum is $\frac{2}{\lambda r}+2 \frac{(2 n+3)^{2}}{L\left(\mathcal{U}_{0}\right)}+\frac{2}{\lambda r}+2 \frac{(2 n+3)^{2}}{L\left(\mathcal{U}_{1}\right)}$-Lipschitz. Hence in this case it has Lipschitz constant equal to $2 \frac{2}{\lambda r}+\frac{(2 n+3)^{2}}{L\left(\mathcal{U}_{0}\right)}+\frac{(2 n+3)^{2}}{L\left(\mathcal{U}_{1}\right)}$. So, we conclude that $\phi_{1}$ is $\lambda_{1}$-Lipschitz, where $\lambda_{1}=\max \left\{c_{n} \sqrt{2}\left(\max \left\{\frac{(2 n+3)^{2}}{L\left(\mathcal{U}_{0}\right)}, \frac{2}{\lambda r}\right\}\right), 2 \frac{2}{\lambda r}+2 \frac{(2 n+3)^{2}}{L\left(\mathcal{U}_{0}\right)}+2 \frac{(2 n+3)^{2}}{L\left(\mathcal{U}_{1}\right)}\right\}$.

Similarly, assuming $\phi_{p-1}$ is $\lambda_{p-1}$-Lipschitz, we consider

$$
\phi_{p}(x)= \begin{cases}q_{p}\left(\phi_{p-1}(x), 2 t_{p}(x)\right), & \text { if } t_{p}(x) \in\left[0, \frac{1}{2}\right], \\ 2\left(1-t_{p}(x)\right) \psi_{(p-1)}^{(p)} \phi_{p-1}(x)+\left(2 t_{p}(x)-1\right) p_{\mathcal{U}_{p}}(x), & \text { otherwise. }\end{cases}
$$

As before in the top case we see that the map is $c_{n+p-1} \sqrt{2} \max \left\{\lambda_{p-1}, \frac{2}{\lambda r}\right\}$-Lipschitz. In the second case, we apply the Leibnitz rule again to see that the Lipschitz constant is $2 \frac{2}{\lambda r}+2 \lambda_{p-1}+2 \frac{(2 n+3)^{2}}{L\left(\mathcal{U}_{p}\right)}$. Thus, we conclude that $\phi_{p}$ is $\lambda_{p}$-Lipschitz with $\lambda_{p}=\max \left\{c_{n+p-1} \sqrt{2} \max \left\{\lambda_{p-1}, \frac{2}{\lambda r}\right\}, 2 \frac{2}{\lambda r}+2 \lambda_{p-1}+2 \frac{(2 n+3)^{2}}{L\left(\mathcal{U}_{p}\right)}\right\}$. Thus, $\phi$ is Lipschitz, with Lipschitz constant $\lambda_{k}$.

Lemma 2. In the notation of the previous lemma, suppose $\sigma$ and $\tau$ are simplices in $N^{\prime}$, both of which are maximal with respect to containment. Suppose that $\sigma \cap \tau=\varrho$. Then $\left.\phi^{(\tau)}\right|_{\varrho}=\left.\phi^{(\sigma)}\right|_{\varrho}$.

Proof. Suppose that the vertices of $\sigma$ are denoted $v_{0}, \ldots, v_{c}$ and the vertices of $\tau$ are $w_{0}, \ldots, w_{d}$, where the index of the vertex corresponds to the dimension of the cell of which the vertex is the barycenter.

First, we show that for all $x$ mapping to $\varrho, t_{p}^{(\sigma)}(x)=t_{p}^{(\tau)}(x)$ for all $p$. If $\varrho=$ $\left[v_{i_{0}}, \ldots, v_{i_{s}}\right]$ as a subsimplex of $\sigma$, then $\varrho=\left[w_{i_{0}}, \ldots, w_{i_{s}}\right]$ as a subsimplex of $\tau$, since the indices must correspond to dimensions of cells in $N$. Clearly $t_{j}(x)=0$ for all the indices that do not appear in the description of $\varrho$. All the other $t_{i_{j}}$ must agree, as they are defined in terms of distances intrinsic to the simplex $\varrho$.

We prove the lemma by induction on the dimension of $\varrho$. To begin, suppose that $\varrho$ is a point. If $\varrho=v_{p}=w_{p}$, then $t_{p}^{(\sigma)}(x)=1=t_{p}^{(\tau)}(x)$ for any $x$ with $p_{N^{\prime}} f(x)=\varrho$. It is also clear that if $i \neq p$, then $t_{i}^{(\sigma)}(x)=t_{i}^{(\tau)}(x)=0$. Thus, $\phi^{(\sigma)}(x)=$ $q_{p+1}\left(\phi_{p}^{(\sigma)}(x), 0, \ldots, 0\right)$. But $\phi_{p}^{(\sigma)}(x)=p_{\mathcal{U}_{p}}(x)$. On the other hand, when we compute $\phi$ thinking of $\varrho$ as a subsimplex of $\tau$, we see $\phi^{(\tau)}(x)=\left(p_{\mathcal{U}_{p}}(x), 0, \ldots, 0\right)$. Thus, we obtain $\phi^{(\sigma)}(x) \approx p_{\mathcal{U}_{p}}(x) \approx \phi^{(\tau)}(x)$, where $\approx$ denotes the natural identification in the mapping cylinder.

Next, consider $\varrho$ with $\sigma$-vertices $\left\{v_{i_{0}}, \ldots, v_{i_{s}}\right\}$. Since the indices on the vertices agree, the $\tau$-vertices must be $\left\{w_{i_{0}}, \ldots, w_{i_{s}}\right\}$. Applying the definition, we see that $\phi^{(\sigma)}(x)=q_{i_{s}+1}\left(\phi_{i_{s}}^{(\sigma)}(x), 0, \ldots, 0\right)$, where

$$
\begin{aligned}
& \phi_{i_{s}}^{(\sigma)}(x) \\
& \quad= \begin{cases}q_{i_{s}}\left(\phi_{i_{s}-1}^{(\sigma)}(x), 2 t_{i_{s}}(x)\right), & \text { if } t_{i_{s}}(x) \in\left[0, \frac{1}{2}\right], \\
2\left(1-t_{i_{s}}(x)\right) \psi_{\left(i_{s}-1\right)}^{\left(i_{s}\right)} \phi_{i_{s}-1}^{(\sigma)}(x)+\left(2 t_{i_{s}}(x)-1\right) p_{\mathcal{U}_{i_{s}}}(x), & \text { otherwise. }\end{cases}
\end{aligned}
$$


Similarly, we find $\phi^{(\tau)}(x)=q_{i_{s}+1}\left(\phi_{i_{s}}^{(\tau)}(x), 0, \ldots, 0\right)$, where

$$
\begin{aligned}
& \phi_{i_{s}}^{(\tau)}(x) \\
& \quad= \begin{cases}q_{i_{s}}\left(\phi_{i_{s}-1}^{(\tau)}(x), 2 t_{i_{s}}(x)\right), & \text { if } t_{i_{s}}(x) \in\left[0, \frac{1}{2}\right], \\
2\left(1-t_{i_{s}}(x)\right) \psi_{\left(i_{s}-1\right)}^{\left(i_{s}\right)} \phi_{i_{s}-1}^{(\tau)}(x)+\left(2 t_{i_{s}}(x)-1\right) p_{\mathcal{U}_{i_{s}}}(x), & \text { otherwise. }\end{cases}
\end{aligned}
$$

By the inductive hypothesis applied to the simplex whose vertices are $\left\{v_{i_{0}}, \ldots v_{i_{s-1}}\right\}$ and $\left\{w_{i_{0}}, \ldots w_{i_{s-1}}\right\}$, we see that the maps $\phi_{p}^{(\sigma)}$ and $\phi_{p}^{(\tau)}$ agree for all $p<i_{s}$, up to identification in the mapping cylinders. Thus, the maps $\phi^{(\sigma)}$ and $\phi^{(\tau)}$ agree on $\varrho$.

Proposition 4. Let $\epsilon>0$ be given. Suppose $\lambda$ is a constant, $\lambda \geq 1$. Finally, suppose that $r<L\left(\mathcal{U}_{0}\right)<\cdots<L\left(\mathcal{U}_{k}\right)$ in the notation of Lemma 1 , where $r \geq$ $\frac{1}{\epsilon}(2 n+3)^{2} 6^{k} c_{n} c_{n+1} \cdots c_{n+k-1}$. Then, $\phi$ is $\epsilon$-Lipschitz, where the $c_{i}$ are the constants from Proposition 1 .

Proof. Again the proof is a simple computation. For $0 \leq p \leq k$, we show that

$$
\lambda_{p} \leq \frac{(2 n+3)^{2}}{r} \max \left\{c_{n} \sqrt{2}, 6\right\} \cdots \max \left\{c_{n+p-1} \sqrt{2}, 6\right\} .
$$

Then, $\lambda_{k}=\frac{(2 n+3)^{2}}{r} \max \left\{c_{n} \sqrt{2}, 6\right\} \cdots \max \left\{c_{n+k-1} \sqrt{2}, 6\right\}$, and so

$$
\lambda_{k} \leq \frac{\epsilon(2 n+3)^{2} 6^{k-\ell} \sqrt{2}^{\ell} c_{n_{1}} \cdots c_{n_{\ell}}}{6^{k} c_{n} \cdots c_{n+k-1}}
$$

for some $\ell$. Since the maps $q$ restrict to an isometry on $t=0$, we have $c_{i} \geq 1$, and so we see that $\lambda_{k}$ does not exceed $\frac{\epsilon}{(3 \sqrt{2})^{\ell}} \leq \epsilon$.

To prove the claim, we use induction. Let $p=1$. We saw in the proof of Lemma 1 that $\lambda_{1}=\max \left\{c_{n} \sqrt{2} \frac{(2 n+3)^{2}}{r}, c_{n} \sqrt{2} \frac{2}{\lambda r}, 2 \frac{2}{\lambda r}+2 \frac{(2 n+3)^{2}}{L\left(\mathcal{U}_{0}\right)}+2 \frac{(2 n+3)^{2}}{L\left(\mathcal{U}_{1}\right)}\right\}$. This does not exceed $\max \left\{c_{n} \sqrt{2} \frac{(2 n+3)^{2}}{r}, 2 \frac{2}{r}+2 \frac{(2 n+3)^{2}}{r}+2 \frac{(2 n+3)^{2}}{r}\right\} \leq \max \left\{c_{n} \sqrt{2} \frac{(2 n+3)^{2}}{r}, 6 \frac{(2 n+3)^{2}}{r}\right\}$. Thus, $\lambda_{1} \leq \max \left\{c_{n} \sqrt{2}, 6\right\} \frac{(2 n+3)^{2}}{r}$, as desired.

To prove the inductive step, we use the estimate in Lemma 1 :

$$
\begin{aligned}
\lambda_{p} & =\max \left\{c_{n+p-1} \sqrt{2} \max \left\{\lambda_{p-1}, \frac{2}{\lambda r}\right\}, 2 \frac{2}{\lambda r}+2 \lambda_{p-1}+2 \frac{(2 n+3)^{2}}{L\left(\mathcal{U}_{p}\right)}\right\} \\
& \leq \max \left\{c_{n+p-1} \sqrt{2} \lambda_{p-1}, \frac{4}{r}+2 \lambda_{p-1}+\frac{2(2 k+3)^{2}}{r}\right\} \\
& \leq \max \left\{c_{n+p-1} \sqrt{2}, 6\right\} \lambda_{p-1} .
\end{aligned}
$$

The last inequality follows by observing that both $\frac{2}{r}$ and $\frac{(2 n+3)^{2}}{r}$ are not more than $\lambda_{p-1}$.

The next proposition is another technical result that relies heavily on the uniform inequality asdim $\leq n$ for a family of metric spaces. We also need the notion of $d$ saturated union. Let $\mathcal{U}$ and $\mathcal{V}$ be families of subsets of a metric space $X$. Denote by $N_{d}(V ; \mathcal{U})$ the union of $V$ and all $U \in \mathcal{U}$ with $d(V, U) \leq d$. The $d$-saturated union, $\mathcal{V} \cup_{d} \mathcal{U}$, is defined to be the family $\left\{N_{d}(v ; \mathcal{U}) \mid V \in \mathcal{V}\right\} \cup\{U \in \mathcal{U} \mid d(U, V)>$ $d$, for all $V \in \mathcal{V}\}$.

Proposition 5. Let $\left\{F_{\alpha}\right\}$ be a collection of subspaces of the metric space $X$ satisfying $\operatorname{asdim} F_{\alpha} \leq n$ uniformly. Then, for any $m \in \mathbb{Z}$ and for any $L>0$ there is $a$ bound $b$ so that there is a b-bounded cover of $\bigcup_{i=1}^{m} F_{\alpha_{i}}$ with multiplicity $\leq n+1$ and Lebesgue number $\geq L$. 
Proof. First, for each $m$, we prove that the collection of $\left\{\bigcup_{\alpha \in I} F_{\alpha}\right\}_{|I|=m}$ has asymptotic dimension $\leq n$ uniformly.

We proceed inductively, the base case being true by assumption. For any collection of $m$ sets, write $\bigcup_{I} F_{\alpha}$ as $F_{\alpha_{0}} \cup \bigcup_{I^{\prime}} F_{\alpha}$, where $I^{\prime}$ is the index set $I$ with $\alpha_{0}$ removed. Take $d$-disjoint, $R$-bounded families $\mathcal{U}_{0}, \ldots, \mathcal{U}_{n}$ covering $F_{\alpha_{0}}$ and $5 R$ bounded, $r$-bounded families $\mathcal{V}_{0}, \ldots, \mathcal{V}_{n}$ covering $\bigcup_{I^{\prime}} F_{\alpha}$ by the inductive hypothesis. Then, put $\mathcal{W}_{i}=\mathcal{V}_{i} \cup_{d} \mathcal{U}_{i}$, the $d$-saturated union. Then, this family is $d$-disjoint and $r+2(d+R)$-bounded. Since this construction was independent of the indexing set, the family $\left\{\bigcup_{\alpha \in I} F_{\alpha}\right\}_{|I|=m}$ has asymptotic dimension $\leq n$ uniformly.

Now, we prove the assertion of the proposition. Let $L$ be given. Take $d=2 L$. Then, construct the covers $\mathcal{W}_{i}$ as in the preceding paragraph. Thus, $\mathcal{W}_{i}$ are $d$ disjoint and $r+2(d+R)$-bounded. Finally, put $\mathcal{W}=\bigcup_{i=0}^{n} N_{d / 2}\left(\mathcal{W}_{i}\right)$. Then $\mathcal{W}$ covers the union $\left\{\bigcup_{\alpha \in I} F_{\alpha}\right\}_{|I|=m}$, and $\mathcal{W}$ has multiplicity $\leq n+1$ and the Lebesgue number of $\mathcal{W}$ is greater than $d / 2=L$, as desired. Clearly, $b=r+2 R+3 d$ is a uniform bound on the diameters of the elements of $\mathcal{W}$.

We are finally in a position to prove the main result of the paper, our Hurewicztype theorem for asdim.

Theorem 1. Let $f: X \rightarrow Y$ be a Lipschitz map of a geodesic metric space to a metric space. Suppose that for every $R>0,\left\{f^{-1}\left(B_{R}(y)\right)\right\}_{y \in Y}$ satisfies the inequality asdim $\leq n$ uniformly. Then $\operatorname{asdim} X \leq \operatorname{asdim} Y+n$.

Proof. Suppose $\operatorname{asdim} Y \leq k$. For a given $\epsilon>0$ we will construct a uniformly cobounded, $\epsilon$-Lipschitz map $\Phi: X \rightarrow K$ to a uniform simplicial complex of dimension $n+k$.

Suppose that $f$ is $\lambda$-Lipschitz. So that we can apply Proposition 4 we observe that $\lambda$ can be taken to be at least 1. Take $r$ as in Proposition 4 and let $\mathcal{W}$ be a cover of $f(X)$ by uniformly bounded sets with multiplicity $\leq k+1$ whose $\lambda r$-enlargements also have multiplicity $\leq k+1$. (Using the first definition of asymptotic dimension it is not difficult to see that such a cover must exist.)

Since the $W \in \mathcal{W}$ are uniformly bounded, there is an $R>0$ so that for every $W \in \mathcal{W}$ there is a $y_{W} \in f(X)$ so that $N_{\lambda r}(W) \subset B_{R}\left(y_{W}\right)$. Thus, $f^{-1}\left(N_{\lambda r}(W)\right) \subset$ $f^{-1}\left(B_{R}\left(y_{0}\right)\right)$. Since by assumption, asdim $f^{-1}\left(B_{R}(y)\right) \leq n$ uniformly, we conclude that $\operatorname{asdim} f^{-1}\left(N_{\lambda r}(W)\right) \leq n$.

So, for each $W \in \mathcal{W}$, let $\mathcal{U}_{0}^{W}$ denote a family of covers of $f^{-1}\left(N_{\lambda r}(W)\right)$ that are uniformly bounded, have multiplicity $\leq n+1$ and have Lebesgue number $L\left(\mathcal{U}_{0}\right)>r$. Inductively define covers $\mathcal{U}_{i}^{\sigma}$ covering $f^{-1}(\sigma)$ for all $i$-dimensional simplices $\sigma$ in Nerve $\left(N_{\lambda r}(\mathcal{W})\right)$. Insist that the covers be uniformly bounded, have multiplicity $\leq n+1$ and take $L\left(\mathcal{U}_{i}\right)>b\left(\mathcal{U}_{i-1}\right)$. This final condition is possible by Proposition 5 ,

Let $N^{\prime}$ denote the barycentric subdivision of $\operatorname{Nerve}\left(N_{\lambda r}(\mathcal{W})\right)$. Let $\tau$ be a simplex in $\operatorname{Nerve}\left(N_{\lambda r}(\mathcal{W})\right)$ maximal with respect to containment. Let $\tau^{\prime}$ be a simplex in the barycentric subdivision of $\tau$ with dimension equal to that of $\tau$. Then we have covers $\mathcal{U}_{i}$ for the vertices $v_{i}$ of $\tau^{\prime}$. The conditions on the Lebesgue numbers and bounds of the covers mean that there are simplicial maps $\psi_{(i)}^{(j)}: \operatorname{Nerve}\left(\mathcal{U}_{i}\right) \rightarrow \operatorname{Nerve}\left(\mathcal{U}_{j}\right)$ whenever $i<j$.

Apply the lemma to obtain a map $\phi: X_{\tau^{\prime}} \rightarrow K_{\tau^{\prime}}$. The map is $\epsilon$-Lipschitz by Proposition 4 and uniformly cobounded by Lemma 1 . Glue the $K_{\tau^{\prime}}$ using the face relation on $N^{\prime}$. By Lemma 2, the $\phi$ agree along common faces. So by Proposition 
2 they define an $\epsilon$-Lipschitz, uniformly cobounded map $\Phi: X \rightarrow K$. Obviously $\operatorname{dim} K=n+k$.

We immediately obtain a result for groups acting by isometries on trees. This upper bound is the product-type estimate one expects; cf. [2], 14].

Theorem 2. Let $\Gamma$ be a finitely generated group acting on the metric space $X$ by isometries. Fix a point $x_{0} \in X$. Suppose that $\operatorname{asdim} X \leq k$ and $\operatorname{asdim} W_{R}\left(x_{0}\right) \leq n$, for all $R$. Then, $\operatorname{asdim} \Gamma \leq n+k$.

Proof. Fix a finite generating set $S=S^{-1}$ for $\Gamma$. Then, $|\Gamma|_{S}$ is a geodesic metric space. Define the map $\pi: \Gamma \rightarrow X$ by $\pi(\gamma)=\gamma x_{0}$. Put $\lambda=\max \left\{d_{X}\left(s x_{0}, x_{0}\right)\right.$ $s \in S\}$. We claim that the $\pi$ is $\lambda$-Lipschitz. Since the metric space $\Gamma$ is discrete geodesic, it suffices to check the Lipschitz condition on pairs of points at distance 1 from each other. Such a pair is of the form $(\gamma, \gamma s)$, for some $s \in S$. Now,

$$
d_{X}(\pi(\gamma), \pi(\gamma s))=d_{X}\left(\gamma x_{0}, \gamma s x_{0}\right)=d_{X}\left(x_{0}, s x_{0}\right) \leq \lambda .
$$

Thus, $\pi$ is $\lambda$-Lipschitz. So that we can apply Proposition 4, we observe that $\lambda$ can be taken to be at least 1 .

Next, observe that $W_{R}\left(x_{0}\right)=\pi^{-1}\left(B_{R}\left(x_{0}\right)\right)$, and since the action is isometric, $\gamma B_{R}\left(x_{0}\right)=B_{R}\left(\gamma x_{0}\right)$. So, $\pi^{-1}\left(B_{R}\left(\gamma x_{0}\right)\right)=W_{R}\left(\gamma x_{0}\right)$ and $W_{R}\left(x_{0}\right)$ is isometric to $W_{R}\left(\gamma x_{0}\right)$ for all $\gamma \in \Gamma$. Thus, asdim $\pi^{-1}\left(B_{R}(x)\right) \leq n$ uniformly. We apply Theorem 1 to get $\operatorname{asdim} \Gamma \leq n+k$.

In 1, Lemma 1], the first author proved the following result about complexes of groups. Complexes of groups are a natural generalization of the Bass-Serre theory of graphs of groups. Whereas graphs of groups describe groups acting on trees, in the general theory trees are replaced with higher-dimensional analogs of trees called small categories without loops (briefly scwols). There is not always an action associated to a complex of groups, so the theory is not as nice as the Bass-Serre theory. When there is an associated action, the complex of groups is called developable. For more details see [5, Chapter III.C].

Proposition 6. Let $\pi$ be the fundamental group of a complex of groups $G(\mathcal{Y})$ where $\mathcal{Y}$ is finite, the local groups $G_{\sigma}$ are finitely generated and $\operatorname{asdim} G_{\sigma} \leq n$ for all $\sigma$. Fix some $\sigma_{0} \in \mathcal{Y}$. Then for every $R>0$, asdim $W_{R}\left(G_{\sigma_{0}}\right) \leq n$.

Applying Theorem 2 and the previous proposition we immediately obtain the following sharpening of [1, Theorem 3].

Theorem 3. Let $\Gamma$ be the fundamental group of a finite, developable complex of groups corresponding to an action by isometries on the geometric realization of the scwol $\mathcal{X}$. Suppose the local groups are finitely generated and asdim $G_{\sigma} \leq n$. If $\operatorname{asdim}|\mathcal{X}| \leq k$, then $\operatorname{asdim} \Gamma \leq n+k$.

When $\mathcal{X}$ is one dimensional, we recover the main theorem from 3 .

\section{LiPSCHITZ MAPPINGS TO TREES}

We want to generalize the main result of [4] where a formula for the asymptotic dimension of a free product of groups is given.

We will need the following results. 
Proposition 7 ([3, Proposition 1]). For every $K$ and every $\epsilon>0$ there exists a number $\nu=\nu(\epsilon, k)$ such that for every cover $\mathcal{U}$ of a metric space $X$ of order $\leq k+1$ with Lebesgue number $L(\mathcal{U})>\nu$, the canonical projection to the nerve, $p_{\mathcal{U}}: X \rightarrow \operatorname{Nerve}(\mathcal{U})$ is $\epsilon$-Lipschitz.

Lemma 3 ([3, Lemma 1]). Let $A$ be a closed subset of the geodesic metric space $X$. Let $r>8 \epsilon$ and let $\mathcal{V}$ and $\mathcal{U}$ be covers of the $r$-neighborhood $N_{r}(A)$ by uniformly bounded open sets such that $\mathcal{V}$ has order $\leq n+1, N$ erve $(\mathcal{V})$ is orientable, and $L(\mathcal{U})>b(\mathcal{V})>L(\mathcal{V}) \geq \nu\left(\epsilon / 4 c_{n}, n\right)$, where $c_{n}$ is the constant of uniformization from Proposition 1. Then there is an $\epsilon$-Lipschitz map $f: N_{r}(A) \rightarrow M_{g}$ to the mapping cylinder supplied with the uniform metric of a simplicial map $g: \operatorname{Nerve}(\mathcal{V}) \rightarrow \operatorname{Nerve}(\mathcal{U})$ between the nerves such that $f$ is uniformly cobounded, $\left.f\right|_{\partial N_{r}(A)}=q\left(\left.p_{\mathcal{V}}\right|_{\partial N_{r}(A)}, 0\right)$, and $\left.f\right|_{A}=\left.p_{\mathcal{U}}\right|_{A}$, where $p_{\mathcal{U}}: N_{R}(A) \rightarrow N e r v e(\mathcal{U})$ and $p_{\mathcal{V}}: N_{r}(A) \rightarrow \operatorname{Nerve}(\mathcal{V})$ are the canonical projections to the nerves.

To improve the Hurewicz-type estimate for maps to trees we need the notion of asymptotic inductive dimension. Asymptotic inductive dimension, asInd, was defined by the second author in [8] in order to establish connections between $\operatorname{asdim} X$ and Ind $\nu X$, where $\nu X$ is the Higson corona of $X$.

Let $\varphi: X \rightarrow \mathbb{R}$ be a function defined on a metric space $X$. For every $r>0$ let $V_{r}(x)=\sup \left\{|\varphi(y)-\varphi(x)|: y \in B_{r}(x)\right\}$. Such a function $\varphi$ is called slowly oscillating if for every $r>0$ and every $\epsilon>0$, there exists a compact set $K \subset X$ so that $V_{r}(x)<\epsilon$ for all $x \in X \backslash K$. Let $\bar{X}$ be the compactification of $X$ corresponding to the family of all continuous bounded slowly oscillating functions. The Higson corona of $X$ is the remainder $\nu X=\bar{X} \backslash X$.

For any subset $A \subset X$ denote by $A^{\prime}$ the trace of $A$ on $\nu X$, i.e. the intersection of the $\bar{X}$-closure of $A$ with $\nu X$.

Let $X$ be a proper metric space. A subset $W \subset X$ is called an asymptotic neighborhood of $A \subset X$ if for any $x_{0}, \lim _{r \rightarrow \infty} d\left(A \backslash B_{r}\left(x_{0}\right), X \backslash W\right)=\infty$.

Two sets $A, B \subset X$ are asymptotically disjoint if for any $x_{0}$,

$$
\lim _{r \rightarrow \infty} d\left(A \backslash B_{r}\left(x_{0}\right), B \backslash B_{r}\left(x_{0}\right)\right)=\infty .
$$

Thus, $A$ and $B$ are asymptotically disjoint precisely when their traces $A^{\prime}$ and $B^{\prime}$ are disjoint.

A subset $C \subset X$ is an asymptotic separator for the asymptotically disjoint sets $A, B \subset X$ if its trace $C^{\prime}$ is a separator for $A^{\prime}$ and $B^{\prime}$. Define asInd $X=-1$ if and only if $X$ is bounded, and otherwise, define asInd $X \leq n$ if for any asymptotically disjoint $A$ and $B$ in $X$ there exists an asymptotic separator $C$ with asInd $C \leq n-1$.

Theorem ([8, Theorem 3]). Let $X$ be a proper metric space with bounded geometry and suppose that $\operatorname{asdim} X$ is positive and finite. Then $\operatorname{asInd} X=\operatorname{asdim} X$.

There is a small problem with bounded sets. If $K$ is a bounded set asInd $K=-1$, whereas $\operatorname{asdim} K=0$. (Note that there are unbounded sets, for example $\left\{2^{n}\right\} \subset \mathbb{Z}$, with asdim zero.) In any case, for metric spaces $X$ with bounded geometry, we have asInd $X \leq \operatorname{asdim} X$; see [10].

Theorem 4. Let $f: X \rightarrow T$ be a Lipschitz map of the geodesic metric space $X$ with bounded geometry to a tree. Suppose that for any disjoint bounded sets $W$ and $W^{\prime}$ in $T$, the sets $f^{-1}(W)$ and $f^{-1}\left(W^{\prime}\right)$ are asymptotically disjoint. Suppose that for every $R>0$, the family $\left\{B_{R}(v)\right\}_{v \in T}$ satisfies the inequality $\operatorname{asdim} f^{-1}\left(B_{R}(v)\right) \leq n$ 
uniformly in $v \in T$, with $n \geq 1$. Then $\operatorname{asdim} X \leq n$. Moreover if $f^{-1}\left(B_{R}(v)\right)=n$ for some $v$ and $R$, then $\operatorname{asdim} X=n$.

Proof. The stronger statement follows since $f^{-1}\left(B_{R}(v)\right) \subset X$. So, it suffices to show $\operatorname{asdim} X \leq n$. Suppose $f$ is $\lambda$-Lipschitz. Given $\epsilon>0$ we construct an $\epsilon$-Lipschitz, uniformly cobounded map $\psi: X \rightarrow K$ to a uniform polyhedron of dimension $n$.

Let $c_{n-1}$ be the constant of uniformization from Proposition [1. Take $\nu=$ $\nu\left(\epsilon / 4 c_{n-1}, n-1\right)$ and let $r>\max \{\nu, 8 / \epsilon\}$. Take a cover $\mathcal{W}$ of $T$ by disjoint sets so that the $\lambda r$-enlargement and the $2 \lambda r$-enlargement both have order 2 .

Since the $W \in \mathcal{W}$ are uniformly bounded there is an $R>0$ so that for each $W \in$ $\mathcal{W}$ there is a $v_{W} \in T$ so that $N_{2 \lambda r}(W) \subset B_{R}\left(v_{W}\right)$. Since $\operatorname{asdim} f^{-1}\left(B_{R}\left(v_{W}\right)\right) \leq n$ uniformly, asdim $f^{-1}\left(N_{2 \lambda r}(W)\right) \leq n$ uniformly.

Consider a pair $W \neq W^{\prime}$ for which $N_{\lambda r}(W) \cap N_{\lambda r}\left(W^{\prime}\right) \neq \emptyset$. By the finite union theorem, $\operatorname{asdim}\left[f^{-1}\left(N_{\lambda r}(W)\right) \cup f^{-1}\left(N_{\lambda r}\left(W^{\prime}\right)\right)\right] \leq n$, so

$$
\operatorname{asInd}\left[f^{-1}\left(N_{\lambda r}(W)\right) \cup f^{-1}\left(N_{\lambda r}\left(W^{\prime}\right)\right)\right] \leq n .
$$

Since $f^{-1}(W)$ and $f^{-1}\left(W^{\prime}\right)$ are asymptotically disjoint, there is an asymptotic separator $A_{e}$ separating them in $f^{-1}\left(N_{\lambda r}(W)\right) \cup f^{-1}\left(N_{\lambda r}\left(W^{\prime}\right)\right)$, with asInd $A_{e} \leq$ $n-1$. (Here, the subscript $e$ refers to the edge $e=\left[W, W^{\prime}\right]$ in the nerve of $N_{\lambda r}(\mathcal{W})$.) Thus, $\operatorname{asdim} A_{e} \leq n-1$.

As $N_{r}\left(A_{e}\right)$ is coarsely isometric to $A_{e}$ we have $\operatorname{asdim} N_{r}\left(A_{e}\right) \leq n-1$. For each edge, let $\mathcal{V}_{e}$ be a uniformly bounded cover of $N_{r}\left(A_{e}\right)$ with multiplicity $\leq n$ and with Lebesgue number $L>r$. For each $W$ cover $f^{-1}\left(N_{2 \lambda r}(W)\right)$ by uniformly bounded sets with multiplicity $\leq n+1$ and with Lebesgue number greater than $\max \left\{b\left(\mathcal{V}_{e}\right) \mid\right.$ $W \in e\}$, where $b\left(\mathcal{V}_{e}\right)$ is an upper bound on the diameters of the sets in $\mathcal{V}_{e}$. Since $X$ is assumed to have bounded geometry, this maximum exists.

The conditions on the Lebesgue numbers along with the fact that $N_{r}\left(A_{e}\right) \subset$ $f^{-1}\left(N_{2 \lambda r}(W)\right) \cap f^{-1}\left(N_{2 \lambda r}\left(W^{\prime}\right)\right)$ guarantee that there exist simplicial maps $g_{W}$ : $\operatorname{Nerve}\left(\mathcal{V}_{e}\right) \rightarrow \operatorname{Nerve}\left(\mathcal{U}_{W}\right)$ and $g_{W^{\prime}}: \operatorname{Nerve}\left(\mathcal{V}_{e}\right) \rightarrow \operatorname{Nerve}\left(\mathcal{U}_{W^{\prime}}\right)$. Take $M_{e, W}$ and $M_{e, W^{\prime}}$ to be the uniform mapping cylinders of the maps $g_{W}$ and $g_{W^{\prime}}$, respectively.

As $r>8 / \epsilon$, we may apply Lemma 3 to $A_{e} \subset \Gamma$ and the covers to obtain $\epsilon$ Lipschitz maps $h_{e, W}: N_{r}\left(A_{e}\right) \rightarrow M_{e, W}$ and $h_{e, W^{\prime}}: N_{r}\left(A_{e}\right) \rightarrow M_{e, W^{\prime}}$ to the uniform mapping cylinders.

For each $W \in \mathcal{W}$, construct a uniformly cobounded $\epsilon$-Lipschitz map $\phi_{W}$ : $f^{-1}\left(N_{2 \lambda r}(W)\right) \rightarrow K_{W}$ to the uniform $n$-dimensional simplicial complex $K_{W}$ by taking the natural projection to the nerve of $\mathcal{V}_{W}$. Such a mapping exists since $r>\nu$, by Proposition 7

We note that the $N_{r}\left(A_{e}\right)$ are disjoint for distinct edges in the nerve. Thus, for each $W \in \mathcal{W}$ define $\psi_{W}: \pi^{-1}\left(N_{2 \lambda r}(W)\right) \rightarrow K_{W} \bigcup_{W \in e} M_{e, W}=L_{W}$ to the uniform complex $L_{W}$, with mapping cylinders attached as the union of the map $\phi_{W}$ restricted to $f^{-1}\left(N_{2 \lambda r}(W)\right) \backslash \bigcup_{W \in e} N_{r}\left(A_{e}\right)$ and the restrictions of $h_{e, W}$ to $N_{r}\left(A_{e}\right) \cap f^{-1}\left(N_{2 \lambda r}(W)\right)$, for all edges $e$ in $\operatorname{Nerve}\left(N_{\lambda r}(\mathcal{W})\right)$ that contain $W$ as a vertex.

We construct $K$ by gluing together the $L_{W}$. Clearly, the dimension of $K$ is at most $n$. The maps $\psi_{W}: \Gamma \rightarrow K$ agree on the common parts $A_{e}$ so they define a map $\psi: X \rightarrow K$. The map $\psi$ is $\epsilon$-Lipschitz by Proposition 2, and uniformly cobounded by Lemma 3 .

What follows is a natural generalization of the combinatorial structure of amalgamated free products studied in 2 . 
Let $X$ and $Y$ be pointed metric spaces. Define a metric space $X \hat{*} Y$ to be the metric space whose elements are alternating words formed from the alphabet $X \backslash\left\{x_{0}\right\} \sqcup Y \backslash\left\{y_{0}\right\}$. Set $x_{0}=y_{0}=\tilde{e}$. Define a norm by the following rule: $\|z\|=$ $0 \Longleftrightarrow z=\tilde{e}$, and $\left\|x_{1} y_{1} \cdots x_{r} y_{r}\right\|=\sum_{i} d_{X}\left(x_{i}, x_{0}\right)+d_{Y}\left(y_{i}, y_{0}\right)$, where we allow $x_{1}=x_{0}$ or $y_{r}=y_{0}$. To define the metric, let $z, z^{\prime}$ be words in $X \hat{*} Y$. Write $z=u v$ and $z^{\prime}=u v^{\prime}$, so that $u$ is a common beginning, which we allow to be $\tilde{e}$. Then, $d\left(z, z^{\prime}\right)=\|v\|+\left\|v^{\prime}\right\|$. Observe that if $X$ and $Y$ are discrete metric spaces with bounded geometry, then so is $X \hat{*} Y$.

We will need the following union theorems in the next proposition. Both are taken from [2].

Theorem (Infinite Union Theorem). Let $X_{\alpha}$ be a family of subsets of the metric space $X$ satisfying the inequality asdim $X_{\alpha} \leq n$ uniformly. Suppose that for every $r>0$ there exists a $Y_{r} \subset X$ so that asdim $Y_{r} \leq n$ and the family $\left\{X_{\alpha} \backslash Y_{r}\right\}$ is $r$-disjoint. Then, asdim $\bigcup_{\alpha} X_{\alpha} \leq n$.

By taking the family to consist of two sets $A$ and $B$ and taking $Y_{r}=B$ for each $r$ we immediately obtain the following Finite Union Theorem as a corollary.

Theorem (Finite Union Theorem). Let $A$ and $B$ be subsets of a metric space $X$. Then, $\operatorname{asdim} A \cup B \leq \max \{\operatorname{asdim} A, \operatorname{asdim} B\}$.

Proposition 8. Let $(X Y)^{m}$ denote the subset $X Y \cdots X Y \subset X \hat{*} Y$. Suppose that $\operatorname{asdim} X \leq n$ and $\operatorname{asdim} Y \leq n$. Then $\operatorname{asdim}(X Y)^{m} \leq n$ for all $m$.

Proof. Let $w \in X \hat{*} Y$ be a word. Put $\ell(w)$ equal to the length of $w$, i.e. $k$ where $w=z_{1} \cdots z_{k}$ and the $z_{i}$ alternate, coming from $X$ and $Y$. Put $P_{k}=\{w \mid \ell(w)=k\}$. Denote by $P_{k}^{X}$ the set $\left\{w \in P_{k} \mid w_{\ell(w)} \in X\right\}$. Similarly, put $P_{k}^{Y}=\left\{w \in P_{k} \mid\right.$ $\left.w_{\ell(w)} \in Y\right\}$. Since $(X Y)^{m} \subset \bigcup_{k=1}^{2 m} P_{k}$, by the Finite Union Theorem, it suffices to show that $\operatorname{asdim} P_{k} \leq n$ for all $k$.

We proceed inductively. If $k=1$, then $P_{k}=X \cup Y$, so by the finite union theorem asdim $P_{1} \leq n$. Obviously, $P_{k+1}^{X} \subset P_{k}^{Y} X$ and $P_{k+1}^{Y} \subset P_{k}^{X} Y$. We show that $\operatorname{asdim} P_{k+1}^{X} \leq n$. The other case is similar. Put $C_{r}=P_{k}^{Y} B_{r}^{X}\left(x_{0}\right)$, where $B_{r}^{X}\left(x_{0}\right)$ is the $r$-ball around $x_{0}$ in $X$. Then, $C_{r} \subset N_{r}^{X \hat{*} Y}\left(P_{k}\right)$, so $C_{r}$ is coarsely isometric to $P_{k}$. Applying the inductive hypothesis, we conclude that $\operatorname{asdim} C_{r} \leq n$.

Next, consider the families $z X$, where $z \in P_{k}^{Y}$. Clearly if $z \neq z^{\prime}$, then $d\left(z x, z^{\prime} x^{\prime}\right)>$ $\|x\|+\left\|x^{\prime}\right\|$. Thus, $\left\{z X \backslash C_{r}\right\}$ is an $r$ disjoint family. Next, since for every $z, x \mapsto z x$ is an isometry in $X \hat{*} Y$, the families $z X$ are isometric. Next, as $z X$ is coarsely isometric to $X$ for all $z$, we conclude that $\operatorname{asdim} z X \leq n$ uniformly. By the infinite union theorem, we conclude that asdim $P_{k+1} \leq n$.

Obviously the result of the previous theorem also holds for subsets of the form $(Y X)^{m}$.

There is a natural tree, $T$, associated to $X \hat{*} Y$. Define the vertices of $T$ to be formal cosets $u X$ and $v Y$, where $u$ and $v$ are words in $X \hat{*} Y$. Connect the vertices $u X$ and $v Y$ by an edge if either $u x=v$ or $v y=u$ for some $x \in X$ or some $y \in Y$.

Proposition 9. As defined above, $T$ is a tree.

Proof. Obviously $T$ is connected: given two vertices one can find a path connecting them by starting at either of the root vertices $x_{0} Y$ or $y_{0} X$. Next if there were a circuit, say $u X=u x_{1} y_{1} \cdots x_{r} y_{r} X$, then this would mean that there exist $x, x^{\prime} \in X$ 
for which $d\left(x, x_{1} y_{1} \cdots x_{r} y_{r} x^{\prime}\right)=0$. But $d\left(x, x_{1} y_{1} \cdots x_{r} y_{r} x^{\prime}\right) \geq \sum_{i=2}^{r} d\left(x_{0}, x_{i}\right)+$ $\sum_{i=1}^{r} d\left(y_{0}, y_{i}\right)$. For this to be zero, we need all $x_{i}=\tilde{e}$ and $y_{i}=\tilde{e}$.

Theorem 5. Let $X$ and $Y$ be discrete pointed metric spaces with bounded geometry, $\operatorname{asdim} X=n$, and $\operatorname{asdim} Y \leq n$, where $n>0$. Then, $\operatorname{asdim} X \hat{*} Y=n$.

Proof. By Theorem 4 we must find a Lipschitz map to a tree, show that bounded disjoint sets in the tree lift to asymptotically disjoint sets in $X \hat{*} Y$ and that for every $R, \operatorname{asdim} f^{-1}\left(B_{R}(v)\right) \leq n$ for all $v \in T$.

Take $T$ as above, and define $f: X \hat{*} Y \rightarrow T$ by $f(u)=u X$. Let $u, v \in X \hat{*} Y$ with common part $w$. Then $u=w u^{\prime}$ and $v=w v^{\prime}$, and then $d(u, v)=\left\|u^{\prime}\right\|+\left\|v^{\prime}\right\| \geq$ $\ell\left(u^{\prime}\right)+\ell\left(v^{\prime}\right)$, where $\ell(t)$ is the length of $t$. Since

$$
d(f(u), f(v))=d(u X, v X)=d\left(u^{\prime} X, v^{\prime} X\right)=\ell\left(u^{\prime}\right)+\ell\left(v^{\prime}\right),
$$

we conclude that $f$ is 1-Lipschitz.

Next, observe that if $u X$ and $v X$ are distinct vertices of $T$, then $d\left(u x, v x^{\prime}\right) \geq$ $\|x\|+\left\|x^{\prime}\right\|$. Let $W$ and $W^{\prime}$ be disjoint bounded subsets of $T$. Then, the sets $\left\{u x_{0}: u X \in W\right\}$ and $\left\{v x_{0}: v X \in W^{\prime}\right\}$ are bounded. Let $t_{0} X \in T$ be given and take $r$ so large that $B_{r / 2}\left(t_{0} X\right) \subset X$ contains $\left\{u x_{0}: u X \in W\right\} \cup\left\{v x_{0}: v X \in W^{\prime}\right\}$. Then, $d\left(f^{-1}(W) \backslash B_{r}\left(t_{0} X\right), f^{-1}\left(W^{\prime}\right) \backslash B_{r}\left(t_{0} X\right)\right) \geq r$. Thus, $f^{-1}(W)$ and $f^{-1}\left(W^{\prime}\right)$ are asymptotically disjoint.

Finally, it is easy to see that $f^{-1}\left(B_{R}(v X)\right) \subset v X(Y X)^{R}$, and so, by Proposition 8. $\operatorname{asdim} B_{R}(v X) \leq n$.

Theorem 6. Let $A$ and $B$ be finitely generated groups with finite asymptotic dimension. Let $C$ be a common subgroup. Then $\operatorname{asdim} A *_{C} B \leq \operatorname{asdim} C+$ $\max \{\operatorname{asdim} A / C, \operatorname{asdim} B / C, 1\}$.

Remark. This estimate is not always an improvement over the previously known estimate $\operatorname{asdim} A *_{C} B \leq 1+\max \{\operatorname{asdim} A, \operatorname{asdim} B\}$ (see [3]), since there is no way to give an upper bound on $\operatorname{asdim} A / C$ in terms of $\operatorname{asdim} A$ and $\operatorname{asdim} C$. In particular, Thompson's group $F$ is a two generator group, and hence is a quotient of $\mathbb{F}_{2}$; but asdim $F=\infty$ (as it contains a copy of $\mathbb{Z}^{n}$ for each $n$ ), whereas asdim $\mathbb{F}_{2}=1$.

Proof. It is well known that every element $x \in A *_{C} B$ admits a unique normal presentation $c \bar{x}_{1} \bar{x}_{2} \cdots \bar{x}_{k}$, where $c \in C$ and $\bar{x}_{i}=C x_{i}$ are non-trivial alternating cosets of $C$ in $A$ or $B$ and $x=c x_{1} \cdots x_{k}$. Given a metric on $A$ and $B$ we define a metric on $C \backslash A$ and $C \backslash B$ by $\bar{d}(C x, C y)=d_{A *_{C} B}(x, C y)$. Thus, we can consider the metric space $(C \backslash A) \hat{*}(C \backslash B)$, where the common point is $\tilde{e}$.

Define a map $\phi: A *_{C} B \rightarrow(C \backslash A) \hat{*}(C \backslash B)$ by defining $\phi(e)=\tilde{e}$ and $\phi(x)=$ $x_{1} \cdots x_{k}$, where $x=c x_{1} \cdots x_{k}$ is the normal presentation of $x$. We claim that $\phi$ is 1-Lipschitz. Since $A *_{C} B$ is a discrete geodesic metric space, it suffices to check the Lipschitz condition on pairs of the form $(x, x s)$, where $s$ is in the generating set $S$. The normal presentation of $x s$ will be either $c \bar{x}_{1} \cdots \bar{x}_{k} s$, or $c \bar{x}_{1} \cdots \bar{x}_{k} \bar{s}$. In the first case, $d(\phi(x), \phi(x s))=\bar{d}\left(C x_{k}, C x_{k} s\right) \leq d\left(x_{k}, x_{k} s\right)=1$. In the second, $d(\phi(x), \phi(x s))=\bar{d}(C, C s) \leq 1$.

By Theorem 5, $\operatorname{asdim}(C \backslash A) \hat{*}(C \backslash B) \leq \max \{\operatorname{asdim}(C \backslash A), \operatorname{asdim}(C \backslash B), 1\}$.

Consider $\phi^{-1}\left(B_{2 R}(\tilde{e})\right)$. First, observe that $B_{2 R}(\tilde{e})$ consists of alternating words $x_{1} x_{2} \cdots x_{k}$, where the $x_{i}$ alternate between $C \backslash A$ and $C \backslash B$, and $\left\|x_{1} \cdots x_{k}\right\| \leq 2 R$. Thus, $\phi^{-1}\left(B_{2 R}(\tilde{e})\right) \subset \bigcup_{\|w\| \leq 2 R} C w$. Since $(C \backslash A) \hat{*}(C \backslash A)$ has bounded geometry, this is a finite union. Applying the finite union theorem we see, $\operatorname{asdim} \phi^{-1}\left(B_{2 R}(\tilde{e})\right) \leq$ 
$\operatorname{asdim} \bigcup_{w} C w \leq \max \{\operatorname{asdim} C w:\|w\| \leq 2 R\}$. But, since $C w$ is coarsely isometric to $C$, we obtain $\operatorname{asdim} \phi^{-1}\left(B_{2 R}(\tilde{e})\right) \leq \operatorname{asdim} C$.

To get the inequality asdim $\phi^{-1}\left(B_{R}(x)\right) \leq \operatorname{asdim} C$ uniformly, we appeal to Proposition 1 of [2], which says that asdim $F_{\alpha} \leq n$ uniformly if there exist 1Lipschitz injective maps $f_{\alpha}: F_{\alpha} \rightarrow X$ to a metric spaces with bounded geometry and $\operatorname{asdim} X \leq n$.

For each $x \in(C \backslash A) \hat{*}(C \backslash B)$, write $x=\omega x^{\prime}$ where either $\left\|x^{\prime}\right\|=R$ or else $\omega=\tilde{e}$. Suppose $y \in B_{R}(x)$. Then, let $z$ be the common part of $x$ and $y$, so that $x=z x^{\prime \prime}$ and $y=z y^{\prime \prime}$. Then, $d\left(x^{\prime \prime}, y^{\prime \prime}\right) \leq R$, and since $x^{\prime \prime}$ and $y^{\prime \prime}$ have no common beginning, we conclude that $\left\|x^{\prime \prime}\right\| \leq R$. Thus, $z=\omega z^{\prime}$. Hence, $y=\omega z^{\prime} y^{\prime \prime}$, where $\left\|z^{\prime}\right\| \leq R$ and $\left\|y^{\prime \prime}\right\| \leq R$. We conclude that $y \in B_{2 R}(\omega)$.

Let $f_{x}: \phi^{-1}\left(B_{R}(x)\right) \rightarrow \phi^{-1}\left(B_{2 R}(\tilde{e})\right)$ be defined by $y \mapsto y^{\prime \prime}$, where $y^{\prime \prime}$ is the word $y$ with the beginning part $\omega$ removed from it. Then $f_{x}$ is an isometry into $\phi^{-1}\left(B_{2 R}(\tilde{e})\right)$, which is a bounded geometry space with $\operatorname{asdim} \phi^{-1}\left(B_{2 R}(\tilde{e})\right) \leq$ $\operatorname{asdim} C$.

Thus, by the Hurewicz theorem, we have the desired estimate.

\section{An EXTENSION THEOREM FOR ASDIM}

Although it was known for some time (see 2]) that extensions of groups with finite asymptotic dimension had finite asymptotic dimension, the Hurewicz-type theorem for group actions, Theorem 2, allows us to give a sharp upper bound estimate for the dimension.

Theorem 7. Let $\phi: G \rightarrow H$ be a surjective homomorphism of a finitely generated group with kernel $K$. Suppose that $\operatorname{asdim} H \leq n$ and $\operatorname{asdim} K \leq k$. Then, $\operatorname{asdim} G \leq$ $n+k$.

Proof. Let $S$ be a finite generating set for $G$, and take the set $\phi(S)$ as a generating set for $H$. We consider $G$ and $H$ in the left-invariant word metric. The group $G$ acts on $H$ by isometries according to the rule $g . h=\phi(g) h$.

We claim that $W_{R}(e)=N_{R}(K)$, where $e$ is the identity element. Indeed, if $d_{S}(g, K) \leq R$, then $d_{\phi(S)}(\phi(g), e) \leq R$. On the other hand, if $g \in W_{R}(e)$, then $\|\phi(g)\|_{\phi(S)} \leq R$. Let $\phi(g)=\phi\left(s_{i_{1}}\right) \cdots \phi\left(s_{i_{k}}\right)$, where $s_{i_{j}} \in S$, and $k \leq R$. Then, $g s_{i_{k}}^{-1} \cdots s_{i_{1}}^{-1} \in K$ and $d_{S}\left(g, g s_{i_{k}}^{-1} \cdots s_{i_{1}}^{-1}\right)=k \leq R$.

Since $N_{R}(K)$ is coarsely equivalent to $K, \operatorname{asdim} N_{R}(K) \leq k$, and the result follows from the theorem.

Using the extension theorem we can prove the following form of Theorem 6 very easily.

Proposition 10. Let $C \triangleleft A$ and $C \triangleleft B$, where $A$ and $B$ are finitely generated groups with finite asdim. Then $\operatorname{asdim} A *_{C} B \leq \operatorname{asdim} C+\max \{\operatorname{asdim} C \backslash A, \operatorname{asdim} C \backslash B, 1\}$.

Proof. There is a natural surjection of groups $A *_{C} B \rightarrow(C \backslash A) *(C \backslash B)$ with kernel $C$. By the extension theorem, $\operatorname{asdim} A *_{C} B \leq \operatorname{asdim} C+\operatorname{asdim}(C \backslash A) *(C \backslash B)$. Applying the formula for the asdim of a free product from [4], we get asdim $A{ }_{C} B \leq$ $\operatorname{asdim} C+\max \{\operatorname{asdim} C \backslash A, \operatorname{asdim} C \backslash B, 1\}$.

Recall that a group $G$ is called polycyclic if there exists a sequence of subgroups $\{1\}=G_{0} \subset G_{1} \subset \cdots \subset G_{n}=G$ such that each $G_{i} \triangleleft G_{i+1}$ and $G_{i+1} / G_{i}$ is cyclic. The Hirsch length of $G$, denoted $h(G)$, is the number of factors $G_{i+1} / G_{i}$ isomorphic to $\mathbb{Z}$. 
Theorem 8. Let $\Gamma$ be a finitely generated polycyclic group. Then asdim $\Gamma \leq h(\Gamma)$.

Proof. Denote the sequence of subgroups satisfying the polycyclic condition by $\{1\}=\Gamma_{0} \subset \Gamma_{1} \subset \cdots \subset \Gamma_{n}=\Gamma$. Then, by Theorem 7 , we have

$$
\begin{aligned}
\operatorname{asdim} \Gamma & \leq \operatorname{asdim} \Gamma_{n} / \Gamma_{n-1}+\Gamma_{n-1} \\
& \leq \operatorname{asdim} \Gamma_{n} / \Gamma_{n-1}+\operatorname{asdim} \Gamma_{n-1} / \Gamma_{n-2}+\operatorname{asdim} \Gamma_{n-2} \\
& \vdots \\
& \leq \operatorname{asdim} \Gamma_{n} / \Gamma_{n-1}+\cdots+\operatorname{asdim} \Gamma_{1} / \Gamma_{0}+\operatorname{asdim} \Gamma_{0} .
\end{aligned}
$$

Since asdim $\Gamma_{i+1} / \Gamma_{i}$ is only positive if $\Gamma_{i+1} / \Gamma_{i}$ is isomorphic to $\mathbb{Z}$, and since in this case $\operatorname{asdim} \Gamma_{i+1} / \Gamma_{i}=1$, we conclude that $\operatorname{asdim} \Gamma \leq h(\Gamma)$.

Since every finitely generated nilpotent group is polycyclic, we immediately obtain the following result.

Corollary 9. Let $\Gamma$ be a finitely generated nilpotent group. Then $\operatorname{asdim} \Gamma \leq h(\Gamma)$.

Corollary 9 can be extended to nilpotent Lie groups $N$ if one defines the Hirsch length $h(N)$ as the sum of the number of factors in $\Gamma_{i+1} / \Gamma_{i}$ isomorphic to $\mathbb{R}$ for the central series $\left\{\Gamma_{i}\right\}$ of $N$. We take an equivariant metric on $N$ and on the quotients. Then the projection $\Gamma_{i+1} \rightarrow \Gamma_{i+1} / \Gamma_{i}$ is 1-Lipschitz and $\Gamma_{i+1} / \Gamma_{i}$ is coarsely isomorphic to $\mathbb{R}^{n_{i}}$. Then we have

Corollary 10. Let $N$ be a nilpotent Lie group endowed with an equivariant metric. Then $\operatorname{asdim} N \leq h(N)$.

Since $h(N)=\operatorname{dim} N$ for simply connected $N$, we obtain

Corollary 11 ([, Theorem 3.5]). For a simply connected nilpotent Lie group $N$ endowed with an equivariant metric, $\operatorname{asdim} N \leq \operatorname{dim} N$.

Actually in view of [12, Corollary 1.F1] the inequalities in Corollaries 10 and 11] are equalities.

Corollary 11 is the main step in the proof of the following

Theorem 12 ([6]). For a connected Lie group $G$ and its maximal compact subgroup $K$, there is a formula $\operatorname{asdim} G / K=\operatorname{dim} G / K$, where $G / K$ is endowed with a $G$ invariant metric.

This theorem in particular allows us to compute asymptotic dimension of the hyperbolic space $\mathbb{H}^{n}=n$.

Corollary 13. asdim $\mathbb{H}^{n}=n$.

Proof. Take $G=O(n, 1)_{+}$and $K=O(n)$.

This computation can be generalized in the spirit of [14].

Let $(X, d)$ be a metric space. By $\mathcal{H}(X)$ we denote the space of balls in $X$ endowed with the following metric:

$$
\rho\left(B_{t}(x), B_{s}(y)\right)=2 \ln \left(\frac{d(x, y)+\max \{t, s\}}{\sqrt{t s}}\right) .
$$

We note that $\mathcal{H}\left(\mathbb{R}^{n}\right)$ is coarsely equivalent to $\mathbb{H}^{n+1}$ [14, Example 2.60].

We recall that a metric space $X$ with $\operatorname{asdim} X \leq n$ is said to satisfy the Higson property [10] if there exists $C>0$ such that for every $D>0$ there exists a cover $\mathcal{U}$ 
of $X$ with $\operatorname{mesh}(\mathcal{U})<C D$ and such that $\mathcal{U}=\mathcal{U}^{0} \cup \cdots \cup \mathcal{U}^{n}$, where $\mathcal{U}^{0}, \ldots, \mathcal{U}^{n}$ are $D$ disjoint. In [14 $X$ satisfying this condition are said to have asymptotic dimension $\leq n$ of linear type. It is shown in [10] that every metric space of bounded geometry with $\operatorname{asdim} X \leq n$ admits a coarsely equivalent metric with the Higson property. Unfortunately the coarse type of $\mathcal{H}(X)$ depends on a metric on $X$, not only on the coarse class of metrics.

Theorem 14. Suppose that the metric space $(X, d)$ possesses the Higson property. Then $\operatorname{asdim} \mathcal{H}(X)=\operatorname{asdim} X+1$.

Proof. Consider the projection $\pi: \mathcal{H}(X) \rightarrow \mathbb{R}$ defined by $\pi\left(B_{t}(x)\right)=\ln t$ and apply Theorem 1 to it (see [14, Corollary 9.21]).

\section{REFERENCES}

1. G. Bell, Asymptotic properties of groups acting on complexes, Proc. Amer. Math. Soc. 133 (2005), no. 2, 387-396. MR2093059 (2005h:20101)

2. G. Bell and A. Dranishnikov, On asymptotic dimension of groups, Algebr. Geom. Topol. 1 (2001), 57-71. MR:1808331 (2001m:20062)

3. 89-101. MR2034954 (2005b:20078)

4. G. Bell, A. Dranishnikov, and J. Keesling, On a formula for the asymptotic dimension of free products, Submitted, 2004.

5. M. Bridson and A. Haefliger, Metric spaces of non-positive curvature, Springer, 1999. MR.1744486 (2000k:53038)

6. G. Carlsson and B. Goldfarb, On homological coherence of discrete groups, J. Algebra 276 (2004), 502-514. MR2058455 (2005a:20078)

7. A. Dranishnikov, Asymptotic topology, Russian Math. Surveys 55 (2000), no. 6, 71-116. MR.1840358 (2002j:55002)

8. 239-247. MR.1890851 (2003a:54038)

9. A. Dranishnikov and T. Januszkiewicz, Every Coxeter group acts amenably on a compact space, Topology Proc. 24 (1999), 135-141. MR.1802681 (2001k:20082)

10. A. Dranishnikov and M. Zarichnyi, Universal spaces for asymptotic dimension, Topology Appl. 140 (2004), 203-225. MR2074917 (2005e:54032)

11. R. Engelking, Theory of dimensions finite and infinite, Sigma Series in Pure Mathematics, vol. 10, Heldermann Verlag, 1995. MR1363947 (97j:54033)

12. M. Gromov, Asymptotic invariants of infinite groups, Geometric Group Theory, London Math. Soc. Lecture Note Ser. (G. Niblo and M. Roller, eds.), no. 182, 1993. MR1253544 (95m:20041)

13. L. Ji, Asymptotic dimension of arithmetic groups., preprint (2003).

14. J. Roe, Lectures on coarse geometry, University Lecture Series, vol. 31, AMS, 2003. MR:2007488 (2004g:53050)

15. G. Yu, The Novikov conjecture for groups with finite asymptotic dimension, Annals of Mathematics 147 (1998), no. 2, 325-355. MR1626745 (99k:57072)

Department of Mathematics, Penn State University, University Park, Pennsylvania 16802

E-mail address: bell@math.psu.edu

Current address: Mathematical Sciences, University of North Carolina at Greensboro, Greensboro, North Carolina 27402

E-mail address: gcbell@uncg.edu

Department of Mathematics, University of Florida, P.O. Box 118105, Gainesville, FLORIDA 32611-8105

E-mail address: dranish@math.ufl.edu 